\title{
Erratum: Dimensionality and design of isotropic interactions that stabilize honeycomb, square, simple cubic, and diamond lattices [Phys. Rev. X 4, 031049 (2014)]
}

Avni Jain, William Piñeros, Jeffrey R. Errington, and Thomas M. Truskett (Received 6 November 2014; published 20 November 2014)

DOI: 10.1103/PhysRevX.4.049902

Subject Areas: Materials Science, Soft Matter, Statistical Physics

In our paper, there is a sign error in the parameter $\lambda_{1}$ in footnote \#60 which presents the parameters of the optimized potentials, $\varphi_{\mathrm{hc}}$ and $\varphi_{\mathrm{squ}}$. For $\varphi_{\mathrm{hc}}, \lambda_{1}=-0.286436$, and for $\varphi_{\mathrm{squ}}, \lambda_{1}=-0.32989$. There are no changes in any other parameters. 